\title{
BOUND-STATE QUARK AND GLUON CONTRIBUTIONS TO STRUCTURE FUNCTIONS IN QCD'
}

\author{
Stanley J. BRODSKY \\ Stanford Linear Accelemtor Center, \\ Stanford Unuersity. Stanford, California 94309
}

\begin{abstract}
One can distinguish two types of contributions to the quark and gluon structure fuactions of hadrons in quant um chromodyaamics: "intrinsic" contributions, which are due to the direct scattering on the bound-state constituents. and "extrinsic" cont ributions, which are derived from particles created in the collision. In this talk, i discuss several aspects of deep inelastic structure functions in which the bound-state structure of the proton plajs a crucial role: (1) the properties of the intrinsic gluon distribution associated with the proton bound-state wavejunction; (2) the separation of the quark structure function of the proton into intrinsic "bound-valence" and extrinsic "nonvalencer components which takes into account the Pauli principle; (3) the properties and identification of intra usic heary quark structure functions; and (4) a theory of shadowing and anti-shadowing of auclear structure functions. directly related to quark-nucleon interactions and the gluon saturation phenomenon.
\end{abstract}

\section{INTRODUCTION}

The main focus of HERA physics will be the measurements of the quark and gluon structure of the proton. Even at the very small values of $x_{B}$, which can be probed at HERA. the QCD predictions for structure functions still depend strongly on the input quark and gluon distributions associated with the bound-state structure of the proton.

In this talk I will dislinguish two separate contributions to deep inelastic lepton-proton seattering: intrinsic (bound-state) and extrinsic structure functions. The extriasic contributions are created by the virtual strong interactions of the lepton and would be present even if the quark fields of the proton were charge-less. The intrinsic bound-valence quark contributions are due to the elect -on scattering on the quarks described by the proton wavefunction; a complete calculation of these contributions would require solving the bound state problem in QCD. As I shall discuss here, both the Pomeron and leading Reggeon contributions are absent in the bound valesce-quark distributions. The leading Regge contri- butions are thus associated with particles created by the photon-hadron scattering reaction, processes extrinsic to the bound state physics of the target hadron itself.

Despite our confidence that QCD is the correct the. ory of strong interactions, there are very Jew definitive theoretical predictions for the non-perturbative bound state quark and gluon distributions which can be $\mathrm{di}$ rectly derived from the theory, although some constraints on the non-perturbative structure of the proton have been obtained using bag models, quark-diquark schemes, QCD sum rules, non-relativistic quark models, and latice gauge theory.

One new approach, Discretized Light-Cone Quantization, has recently been proposed as a possible way to compute non-perturbative structure functions in gaigh. theory. In this method one attempts to numerically di. agonalize the QCD Hamiltonian quantized on the lightfront in light-cone gauge. One chooses as a basis a complete set of diacrele momentum-space color-singlet free gluon and quark Hamiltonias Fock states satisfying periodic and anti-periodic boundary conditions, tespec.

- Work supported in by the Department of Energy contract DE-AC03-76\$F00515.

Inuted Talk. Presented af the DESY Topical Mteting: Small-x Behavior of Deep Incloster Structure Functions in $Q C D$, Hambung. Germany, May 14-16. 1990.

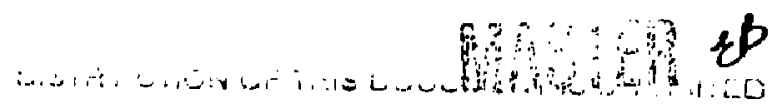


uvely: In principle, the eigenvalues of the full Hamiltonian provide the entire invariant mass spectrum and the corresponding eigenfunctions provide the structure functions and distribution amplitudes peeded for QCD factorization formulas. Thus iar, the main success of DLCQ has been applications to gauge theories in onespace and one-lime dimensions.' For example, the spectrum and structure functions of mesons, baryons, and nuclei in $Q C D(1+1)$ for $S U(3)_{C}$ bave been obtained as a function of mass and coupling constant. Results for the structure function of the lowest mass meson and baryon at weak and strong coupling are shown in Fig. I.

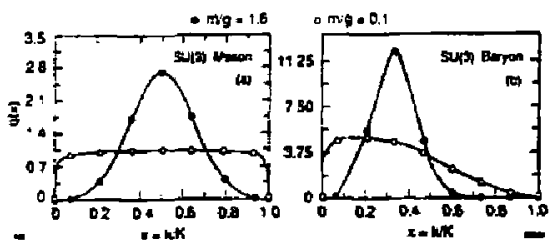

Figure 1. Valence etructure function of the baryon and meson in QCD in one space and ane-time dimension. The results are for one quark flaver and three colors.

The application of DLCQ to gauge theory in threespace and one-time dimensions is a much more challenging computat onal task, but progress has recently been made abtaining the spectrum of QED in the strong conpling domain.

In the following sections, I will discuss a number of general features of bound-state diatributions for intrinsic gluons, bound-valence quarks, and intrinsic heavy-quark states. I will also discuss a new approach to shadowing and anti-shadowing in nuclear structure functions, which analytically relates these phenomena to quark and antiquark nucleon scattering processes.

\section{THE INTRIASIC GLLON DISTRIBLTTJON OF THE PROTON}

Th: gluon distribution of a hadron is often assumed to be radiatively generzed from QCD evolution of the quark structure functions beginning at an initial scale $Q_{0_{0}^{2}}^{2}$ In such a model one assumes that there are no gluons in the hadron at a resolution scale below $Q_{0}$. The evolution is completely incoherent; i.e. each quark in the hadron radiates independently.

However, as can be seen in the light-cone Hamiltonian approsech, the bigher Fock components of a bound state in QCD contain gluons at any resolution scale. The exchange of gluon quants in the bound state generates an interaction potential; the retardation (energydependent) part of the potential contributes to the intrinsic gluon distribution. Notice that the interference diagrams in which gluons are emitted from different quarks are not included in the usual extrinsic gluon distribution computed from the perturbative QCD evolution equations, since in leading twist these contributions only involve a single quark source.

More specificaliy, we can relate the distribution function of intrinsic photons in an atom or intrinsic gluons in a hadron to the hyperfine (spin-dependent) part of the bound state potential since both depend on the exchange of transverse gauge quanta. Each diagram that contributes to the transverse potential has a corresponding cut-diagram in the expression for the distribution function. In the actual calculation, these quantities dif. fer by just a detominator $D$. Thus

$$
\int_{0}^{1} d x G_{g / B}\left(x, Q_{0}^{2}\right)=-\left(\frac{\partial V}{\partial M_{B}^{2}}\right)_{Q g}
$$

where $G_{w / B}$ is the uppolarized distribution function of gunge fields $g$ in the bound atate $B, V$ is the potential due to gluon exchange and self-energy corrections, and 
$M_{B}$ is the bound-state mass. Note that the instantaneous (non-retarded) piece does not depend on $M_{B}$, so it does nol contribute.

In the case of gluons in QCD bound states, we pbtain: $^{3}$

$$
\int_{0}^{1} d x\left[G_{v / p}(x)-G_{v / 4}(x)\right]=-\left\langle\frac{\Delta \partial V}{\partial M_{B}^{2}}\right\rangle_{h f,}
$$

for baryons ( $p$ and $\Delta$ ).

The intrinsic gluon distribution $G_{d / \theta}\left(x, Q_{0}^{2}\right)$ describes the light-cone momentum distribution of gluons associated with the bound-state dynamics of the hadron $H$, in distinction to the extrinsic contributions which are derived from radiative processes or evolution. The intrinsic gluon distribution is derived from the solution of the non-perturbative bound state equation. In the case of quantum electrodynamics, one can readily cajculate the photon distribution in positronium, to fist order in the fine structure constanl $\alpha$. The apalysis involves coherence between amplitudes in which the electron and position couple to the photons. In the infrared limit this coherence in the neutral atom ensures a finite photon distribution.

In the QCD case, the analysis of the intrinsic gluon distribution of a hadron is essentially non-perturbative. However, there are several theoretical constraints which limit its form:

1. In order to insure positivity of fragmentation functions, distribution functions $G_{e / v}(x)$ must behave as an odd or even power of $(1-x)$ at $x \rightarrow I$ according to the relative statistics of $c$ and $b$. Thus the gluon distribution of a nucleon must have the behavior: $G_{\rho / N}(x) \sim(1-x)^{2 k}$ at $x \rightarrow 1$ to ensure correct crossing to the frogmentution function $D_{N / v}(z)$. This result holds individually for each helicity of the gluon and the nucleon.
2. The coupling of quarks to gluons tends to match the aign of the quark helicity to the gluon helicity in the large $x$ limit. We define the belicity-aligned and anti-aligned gluon distribulions: $G^{+}(x)=G_{n / N_{1}}(x)$ and $G^{-}(x)=G_{g 1 / N^{\prime}}(x)$. The gauge theory couplings imply

$$
\lim _{x \rightarrow 1} G^{-}(x) / G^{+}(x) \rightarrow(1-x)^{2}
$$

3. In the low $x$ domein, each of the quarks in the hadron radiate gluone coherently, and one must compute emission of gluons from the quark lines taking into account interference between amplitudes. Define $\Delta G(x)=G^{+}(x)-G^{-}(x)$ and $G(x)=G^{+}(x)+G^{-}(x)$. We find that the asymmetry ratio $\Delta G(x) / G(x)$ vanishes linearly with I; perhaps coincidentally, this is also the prediction from Reggeon exchange." The coefficient at I $\rightarrow 0$ depends on the hadronic wavelunctions; however, for equal partition of the hadion's momentum among its constituents, we show that

$$
\lim _{x \rightarrow 0} \Delta G(x) / G(x) \rightarrow N_{1} x
$$

where $N_{1}$ is the number of valence quarks.

4. In the $x \rightarrow 1 \mathrm{lmit}$, the stuck quark is far off-shel] so that one can use perturbation theory to characterize the threshold dependence of the structure functions. We find for three-quark bound states

$$
\lim _{x \rightarrow 1} G^{+}(x) \rightarrow C(1-x)^{2 N_{4}-2}=C(1-x)^{4}
$$

Thu $G^{-}(x) \rightarrow C(1-x)^{6}$ at $x \sim 1$. This is equivslent to the apectator-counting rule developed in Ref. 7.

We can write down \& simple analytic model for the intrinsic gluon distribution in the nucleon which incor- 
porates all of the above const raints:

$$
\Delta G(x)=\frac{N}{g}\left[5(1-x)^{4}-4(1-x)^{5}-(1-x)^{6}\right]
$$

and

$$
G(x)=\frac{N}{x}\left[5(1-z)^{4}-4(1-z)^{5}+(1-z)^{6}\right]
$$

In this model the momentum fraction carried by intrinsic gluons in the nucleon is $\left(x_{g}\right)=\int_{0}^{1} d_{s x} G(x)=(10 / 21) N$, and the helicity carried by the intrinsic gluons is $\Delta G \equiv$ $\int_{0}^{1} d x \Delta G(x)=7 / 6 N$. The ratio $\Delta G /\left\langle I_{s}\right\rangle=49 / 20$ for the intrinsic gluon distribution is independent of the normalization $\mathbf{N}$. Phenomenological analyses imply that the gluons carry approximately one-half of the proton's momentum: $\left\langle z_{g}, N\right\rangle \simeq 0.5$. We shall assume that this is a good characterization of the intrinsic gluon distribution. The momentum sum rule then implies $N \sim 1$ and $\Delta G \sim 1.2$. In terms of anomalous contributions to the quark spin is concerned, this is a relatively small contribution. However, since $\frac{1}{2} \sum \Delta q+\Delta G+L_{x}=\frac{1}{2}$, a large fraction of the proton's angular momentum is associated with the gluon distribution. A review of the present experimental and cheoretical limits on gluon and quark spin in the nucleon is given in Ref. 8 .

The above equations give model forms for the polarized and unpolarized intrinsic gluon distributions in the nucleon which cake into account coberence at low $x$ and perturbative constraints at high $x$. It is expected that this should be a good characterization of the gluon distribution at the resolution scale $Q_{0}^{2} \simeq M_{0}^{2}$.

It is well-known that the leading power at $z \sim 1$ is increased when CCD evolution is taken into account. The change in power is

$$
\Delta p_{Q}\left(Q^{2}\right)=4 C_{\lambda} \zeta\left(Q^{2}, Q_{0}^{2}\right)=\frac{1}{\pi} \int_{Q_{0}^{2}}^{Q^{2}} \frac{d x^{2}}{\kappa^{2}} \alpha_{d}\left(\kappa^{2}\right),
$$

where $C_{A}=3$ in QCD. For typical values of $Q_{0} \sim$ $1 \mathrm{GeV}, \Lambda_{\text {IS }} \sim 0.2 \mathrm{CeV}$ the change in power is mod- efale: $\Delta p_{0}\left(2 G e V^{2}\right)=0.28, \Delta p_{g}\left(10 G_{t} l^{2}\right)=0.78$ A recenl determination of the unpolarized gluon dis. tribution of the proton at $Q^{2}=2 \mathrm{GeV}^{2}$ using direct photon and deep inelastic data has been given in Ref. 9. The best fit over the interval $0.05 \leq x \leq 0.75$ assum: ing the form $r G\left(x, Q^{2}=2 G e V^{3}\right)=A(J-x)^{\eta_{r}}$ gives $\eta_{g}=3.9 \pm 0.11(+0.8-0.6)$, where the errors in parenthecis allow for systematic unccrtainties. This result is compatible with the prediction $\eta_{g}=4$ for the intrinsic gluon distribution at the bound-state scale, allowing for the increase in the powes due to evolution. HERA experiments could provide a definitive check on the shape and large-x behavior of the gluon structure function.

\section{BOUND VALENCE-QUARK DISTRIBUTIONS}

An important concept in the description of any bound atate is the definition of "valence" constituents. In atomic physics the term "valence electrons" refers to the electrons beyond the closed shells which give an atom its chemical properties. Correspandingly, the term "valence quarks" refers to the quarks which give the bound state hadron its global quantum numbers. In quanturn field theory, the valence quarks appear in each Fock state together with any number of gluons and quark-antiquark puirs; each component thus has the global quan. tum numbers of the hadron.

How can one identify the contribution of the valence quarks of the bound state with the phenomenological structure functions? Traditionally, the distribution function $G_{7 / H}$ has been separaled into "valence" and "sea"

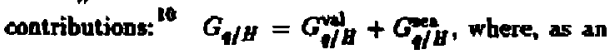
operational definition, one assumes

$$
G_{\sigma / H}^{*}\left(x, Q^{2}\right)=G_{T / B}^{* a}\left(x, Q^{2}\right), \quad(0<x<1),
$$

and thus $G_{G / H}^{\mathrm{v}}\left(\mp, Q^{2}\right)=G_{q / H}\left(x, Q^{2}\right)-G_{T / H}\left(x, Q^{2}\right)$. The assumption of identical quark and anti-quark sea distributions is plausible for the $s$ and $\overline{3}$ quarks in the 
proton. However, in the case of the $u$ and $d$ quark contributions to the sea, anti-symmetrization of identical quarks in the higher Fock states implies non-identical $q$ and $\bar{q}$ sea contributions. This is immediately appar. etit in the case of atcmic physics, where Bethe-Hejtler pair production in the field of an atom does not give symmetric electron and positron distributions since elec. tron capture is blocked in states wherc an atomic electron is already present. Similarly, in QCD, the $q \bar{q}$ pairs which arise from gluon splitting do nol have identi. cal quark and anti-quark sea distributions; contributions from interference diagrams, which arise from the anti-symmetrization of the higher Fock state wavefunctions. must be taken into account. Notice that because of wave-function normalization, the exclusion principle does not afiect the value of conserved charges such as $\int_{0}^{1} d x\left(G_{q / B}(x)-G_{\bar{T} / B}(x)\right)$. Thus even though the conventional separation of valence and sea contributions gires cotrect charge sum rules, it can give a misleading reading of the actual momentum distribution of the valence quarks.

The standard definition also has the difficulty that the derived valence quark distributions are apparently singular in the limit $\mathbf{x} \rightarrow \mathbf{0}$. For example, standard phenomenology indicates that the valence up-quark distribution in the proton behaves as $G_{w / p} \sim x^{-0}$ for amall $x^{10.11}$ where $\alpha_{R} \approx 0.5$. Note that the position $\alpha_{R}$ of,$J$-plase singularities in the forward virtual Compton amplitude are $Q^{2}$-independent, and thus the nonsinglet Reggeon behaviar $F_{2}^{N S}\left(x, Q^{2}\right) \sim x^{1-\alpha n}$ at $x \rightarrow 0$ must be unafiected by QCD evolution. ${ }^{12}$ This implies that quantities that depend on the $(1 / \tau)$ moment of the valence distribution diverge. This is the case for the "sigma Lerm" in current algebra and the $J=0$ fixed pole in Compton scattering. ${ }^{13}$ Furthermore, it has been shown ${ }^{14}$ that the change in mass of the proton when the quark mass is varied in the light-cone Hamiltonian is given by an extension of the Feynman-Hellmann the- orem:

$$
\frac{\partial M_{p}^{2}}{\partial m_{q}^{2}\left(\bar{Q}^{2}\right)}=\int_{D}^{l} \frac{d x}{I} G_{q / p}\left(x, Q^{2}\right)
$$

In principle, this formula allows one to compute the contribution to the proton-neutron mass difference due to the up and down quark masses. However, again, with the standard definition of the valence quark distribution, the integration is undefined at low $x$. Even mors seriously, the expectation value of the light-cone kinetic energy operator

$$
\int_{0}^{1} d x \frac{\left(k_{\perp}^{2}\right)+m^{2}}{x} G_{g / p}(x, Q)
$$

is infinite for valence quarks if one uses the traditional definition. There is no apparent way of associating this divergence of the kinetic energy operator with renormal. ization.

Part of the difficulty witb identifying bound state contributions to the proton structure functions is that many physical processes contribute to the derp inelas. tic leplon-proton cross section: From the perspective of the laboratory or center of mass frame, the virtual photon can scatter out a bound-state quark as in the atomit physics photoelectric process, or the photon can first make a $q \bar{q}$ pair, either of which can intetact in the target. As we emphasize heje, in such pair-production processes, one mast take into account the Pauli principle which forbids creation of a quark in the same statc as one already present in the bound state wavefunction. Thus the lepton interacts with quarks which are both in. trinsic to the proton's bound-state structure, and with quarks which are extrinsic; i.e. created in the electronproton collision itself. Notice that such extrinsic processes would occur in electroproduction even if the va. lence quarks had no charge. Thus much of the phenomena observed in electroproduction at smal! values of 
x, such as Regge behavior, sen distributions associated with phototl-gluon fusion processes, and shadowing in nuclear structure functions should be identified with the extrinsic interactions, father than processes directly connected with the proton's bound-state structure.

Recently, Schmidt and $1^{15}$ bave proposed a new definition of "bound valence-quark" distribution functions that correctly isolates the contribution of the valence constituents which give the hadron its flavor and other global quantum numbers. With this new separation. $G_{q / p}\left(x, Q^{2}\right)=G_{V / p}^{\mathrm{VV}}\left(x, Q^{2}\right)+G_{q / p}^{\mathrm{NV}}\left(x, Q^{2}\right)$, the non-valence quark distributions are identified with the structure fonctions which would be measured if the ra lence quarks of the target hadron had zero electro-weak charge. We can show that with this new definition the bound valence-quark distributions $G_{q / p}^{\mathrm{BV}}\left(x, Q^{2}\right)$ vanish at $I \rightarrow 0$, as expected from the wave function of a boundstate constituent.

In order to construct the bound valence-quark distributions, we imagine a gedanken QCD where, in addition to the usual set of quarks $\{q\}=\{u, d, s, c, b, t\}$, there is another set $\left\{g_{0}\right\}=\left\{t_{0}, d_{0}, s_{0}, c_{0}, b_{0}, t_{0}\right\}$ with the same spin, masses, flavor, color, and other quantum numbers, except that their electromagnetic charges are zero. Let us now consider replacing the target proton $p$ in the lepton-proton scattering experiment by a chargeless proton po which has valence quarks go of zero electromagnetic charge. In this extended QCD the higher Fock wavefunctions of the prolon $p$ and the charge-less proton $p$ both contain $q \bar{q}$ and $g 0 \overline{q 0}$ pairs. As far as the strong QCD interactions are concerned, the physical proIon and the gedonken charge-less proton are equivaleat.

We then define the bound valence-structure function of the proton from the difference between scattering on the physical proton minus the ecattering on the chargeless pioton, in analogy to an "empty target" subtraction:

$$
F_{i}^{B V}\left(x, Q^{2}\right) \equiv F_{i}^{p}\left(x, Q^{2}\right)-F_{i}^{p o}\left(x, Q^{2}\right)
$$

The non-valence distribution is thus $F_{1}^{n}\left(r . Q^{2}\right)=$ $F_{1}^{P_{0}}\left(x, Q^{2}\right)$. Hese the $F_{1}\left(x, Q^{2}\right)(z=1,2$, elc. $)$ arc the leading twist structure functions. The situation just described is similar to the atomic physics case. where in order to coriectly define plioton scaltering from a bound electron, one must subtract the cross sex. tion on the nucleus alone, without that bound electron present. $^{16}$ Physically, the nucleus can scatter photons through virtual pair production, and this contribution bas to be subtracted from the total cross section. In QCD we cannot construct protons without the valenct quarks; thus we need to consider hadrons with chargeless valence constituents.

In order to specifically isolate the bound valence $d$ quark distribution of (he proton $p(u) d)$, we subtrart the deep inelastic cross section on the system po $\left(u u d_{0}\right)$ in which the $d_{0}$ valence quark has normal QCD interar. tions but does not carry electric charge. (Both $p$ and po contain bigher Fock stales with arbitrary number of gluons, $q \bar{q}$, and $q 0 \overline{q 0}$ pairs.) It is clear that the terms as. sociated with $J \approx 1$ Pomeron behavior due to gluon exchange cancel in the difference. We also can show that the Rezgeon terms also cancel, and thus the resulting distribution of bound valence $d$ quarks $G_{d / p}^{B V}\left(x, Q^{2}\right)=$ $\left[\left[F_{2}^{(\operatorname{cod})}\left(x, Q^{2}\right)-F_{2}^{\text {poludo })}\left(x, Q^{2}\right)\right] / \varepsilon_{\mathrm{d}}^{2} \mathrm{x}\right]$ vanishes as $x \rightarrow 0$.

The high $Q^{2}$ virtual pholo-absorption cross section on the proton (lab frame) contains two types of terms: contributions in which a quark in p absorbs the momentum of the virtual photon; and terms in which a $q \bar{q}$ pair is created, but the produced $q$ is in a different quant um state than the quarks already present in the hadron. On the other hand, the cross section for scattering of the virtual photon from the state $p_{0}\left(u u d_{0}\right)$ contains contri. butions that differ from the $p($ urd $)$ case in two important aspects: first the virtual photon can be absorbed only by charged quarks; and in $d \bar{d}$ pair production on the null proton $p_{0}$, the $d$ quark can be produced in any 
state. Thus the difference between the cross sections off $p$ and $p_{0}$ equals a term (analogous to $\sigma_{\text {phoroterire in }}$ atomic physics). in which a $d$ quark in $p$ absorbs the photon momentum. minus a $d \bar{d}$ pair production contribution on po fanalogous to a capture cross section in atomic physics), in which the produced $d$ quark ends up in the same quantum state as the $d$ quark in the original proton state p. ${ }^{17}$ This is shown graphically in Fig. 2.

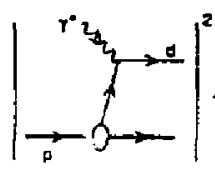

at

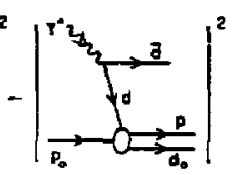

(b)
Figure 2. The bound valence-quark distribution of quark $d$ is calculated from the difference between (a) the croses axtion on the state $P(u u d)$ in which the virtual photon momentum is absorbed by the quart $d$, and (b) the $d \bar{d}$ pair production cross section in the field of the gedanken baryon $p_{0}$ (uudo!, where the produced d quark is captuned in the same state as the $d$ quark in the original proton state $p$

Reggeon behavior in the electroproduction cross section can be understood as due to the appearance of a spectrum of bound $q \bar{q}$ states in the $t$-channel. The summation of such diagrams leads to Reggeon behavior of the deep inelastic structure functions at small $x^{18}$ In the rest system, the virtual photop creates a d $\bar{d}$ pair at a distance proportional to $1 / 2$ before the target. The radiation which occurs over this distance contributes to the physics of the Regzeon bebavior. In the case of the proton target, the $d$-quark, after radiation, cannot appear in the quantum state already occupied by the $d$-quark in the proton because of the Pauli principle. However, the coiresponding contribution is allowed on the po tar. get: in effect, the $d$-quark replaces the $d_{0}$-quark and is captured into a proton. The capture cross section is computed from the amplitude for $\gamma^{4} \mathrm{Pu}_{0} \rightarrow \overrightarrow{\mathrm{d}}^{*} \mathrm{p} \mathrm{d}_{0}{ }^{19}$ As in the corresponding atomic physics analysis, the spectator $d_{0}$ quark in the null target $p_{0}$ is inert and cancels out from the amplicude. Thus we only need to cunsider effectively the (helicity summed) stuared amplitude for $\gamma(u u) \rightarrow \bar{d}^{*} p$. However, as illustrated in FIg. 3 this all plitude. after charge conjugation and rrossing : - u. is equal to the (helicity stimmed) $\gamma^{*} p \rightarrow d^{*}(u u)$ amplit ust. at small $x$. The flux factors for the proton and null pro, ton tatget afe equal.

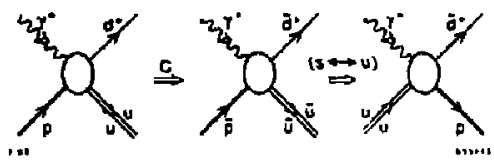

Figure 3 The heliciay-sunimed squared arinplistuds. for (a) $\eta^{*} \nu \rightarrow d(u e)$ is equal, by eharge conjugation. to the helicity-summed squared amplutude for the process (b) $7 \bar{p} \rightarrow \bar{d}(\overline{U T})$, up to a phase. Thus is also equal. by crossing symmetry. to the helicity-summed squared anplitude for (c) $7(u u)-\overline{d p}$, with s and $u$ interchanged.

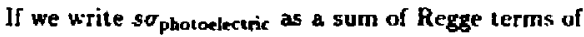
the form $B_{A}|s|^{\alpha_{R}}$, where $a_{R}>0$ then the subtraction of the capture cross section on the null proton will give the net vjrtual photo-absotption cross section as a sum of terms $s \sigma^{B Y}=\sum_{R} \beta_{R}\left(\left.j\right|^{\alpha_{A}}-|u|^{A_{R}}\right)$. If we ignore mass corrections in leading twist, then $s \simeq Q^{*}(1-x)$ ir and $u \simeq-Q^{2} / x$. Thus for small $x$ every Regge tetın is multiplied by a factor $h_{R}=\left(-a_{R}\right)$. For example. for $a_{R}=1 / 2$ (which is the leading even charge-conjugation Reggeon contribution for non-singlet isospin structure functions). $F_{2}^{\text {(wod) }}-F_{2}^{\text {poluwde }} \sim x^{3 / 2}$. The bound valence-quark non-singlet $(l=1)$ distribution thur has leadiug behavior $G_{q / H}^{\mathrm{BV}} \sim J^{1 / 2}$ and vanishes for $\boldsymbol{F} \rightarrow 0$.

We can also understand this result from symmetry considerations. We bave shown from crossing symmetry $G_{q / p}\left(x, Q^{2}\right)-G_{\bar{q} / p}\left(x \cdot Q^{2}\right)-0$ at low $x$. Thus the pterl charge-conjugation Reggeon and Domeron contrjbutions. decouple from the bound valence-quark distributions.

The essential reason why the new definition of the bound valence-quark distribution diffelz from the convetstional definition of valence distibutions is the Pauli 
principle: the anti-symmetrization of the bound state wavefunction for states which contain quarks of identical flavor. As we have shown, this effect plaj's a dynamical role at low $x$, eliminating leading Regge behavior in the bound salence-quark distributions. In the atomic physics case, where therc is no leading Aegge behavior. the analogous application of the Pauli principle leads to analytic consistency with the Kramers-Kronig dispersion relation for Compton scattering on a bound electran. 16

\section{+. NATRINSIC CHARM-QUARK DISTRIBUTIONS}

There are a number of striking anomalies in the data $^{20}$ for charm production which cannot be readily explained by conventional fusion subprocesses.

1. The EMC data ${ }^{21}$ for the charm structure function of the nucleon appears to be too high at large $x_{B y}$.

2. The LEBC bubble chamber data ${ }^{22}$ for charm production in pp collisions indicates an excess of $D$ events at large $\boldsymbol{X}_{\boldsymbol{F}}$. The excess is not associated with $D^{\prime} s$ that contain the pioton's valence quark.

3. The cross section measured by the WA-62 group ${ }^{23}$ for $\Sigma^{-} N \rightarrow \Xi(\operatorname{csu}) X$ is too large and flat at large $X_{F}$.

4. The NA-3 data $^{24}$ for $J / \psi$ production in pionnucleus and proton-nucleus collisions can be represented as tho components: a nomal contribution in the central region which is a most additive in nuclear number that can be accounted for by $g g \rightarrow c \bar{c}$ and $q \bar{q} \rightarrow c \bar{c}$ fusion, and a second "diffractive contribution" which dominates at large $x_{F}$ and is strongly shadowed. This last contribution indicates that high momentum $\bar{c} \bar{c}$ systems are being produced on the surface of the nuclear target.

It is difficult to understand any of these anomolies, particularly the production of high $r F$ charmonium un. less the proton itself has an intrinsic charm contribu. tion $^{25}$ to jts structure function. If these charm quarts are associated with the bound-state equation for the proton, then all the partons tend to have equal velocily: This implies that the heaviest constituents, the intrinkit charm quarks, will take a large fraction of the proton's momenturn. In a hadrotic collision the $c$ and $\bar{c}$ can coalesce to produce a charmonium state with the majority of the proton's momenturn. The EMC charm strurture function data requires a $\mathbf{0 . 3 \%}$ probability for the intrinsic charm Fock state in the nucleon.

According to the hard scattering picture of QCD. production cross sections involying large momentum transfer should factorize and be approximately addi. tive in the nucleon number, $d \sigma_{A}=A^{\alpha}\left(x_{F}, p_{T}\right) d \sigma_{F}$ with $a \sim 1$, up to the small shadowing and antj-shadowing corrections seen in deep inelastic lepton-nucleus scattering. I will return to these effects in the next section.

In the Drel]-Yan process, large mass muon pair production, $\alpha \simeq 1$ for all $x_{F}$ is indeed observed. ${ }^{30}$ However. several experiments on open charm production show ${ }^{20}$ that $\alpha\left(x_{F} \geq 0.2\right) \simeq 0.7 \ldots 0.8$. For small $\mathrm{s} F$, an indireet analysis ${ }^{22}$ comparing different measurements of the total charm production cross section indicates $a(x F \simeq$ $0) \simeq 1$. More detailed data on the nuclear dependence of charm production is arailable from the hadroproduction of $J / \psi$. Here a decrease of a from $a\left(x_{F} \simeq 0\right) \simeq 1$ to $a\left(I_{F} \simeq 0.8\right) \simeq 0.8$ has been seen by several groups. ${ }^{31}$ The analysis of Badier, et al. ${ }^{24}$ is particularly interest. ing. They noted that the production of $J / t$ at large If (up to $x_{F} \simeq 0.8$ ) cannot be explained by the gluon and light guark fusion mechanisms of perturbative QCD, due to the anomalous $A-$ dependence. However, their $x^{-} A \rightarrow J / \psi+X$ data was well reproduced $i$, in addition to hard QCD fusion (with $\alpha=0.97$ ), they included a "diffractive" component of $J / 4$ production at high $x_{F}$ with $a=0.77$ Using their measured $A$-dependence to extract the "diffactive" component, they found that 
(for a pion beam) that the $J / t^{\prime}$ distribution peaks at I $₹ \simeq 0.5$ and dominates the hard scattering $A^{1}$ compo nent for $r \geq 0.6$.

A diffractive contribution to heary quarkonium projuction is consistent with QCD. In high energy hadronnucleus collisions the nucleus may be regarded as a "filter" of the hadronic wave function. ${ }^{28}$ The argument, which relies only on general features such as time dilation, goes as follows. ${ }^{2 t}$ Consider the equal-time Fock stale exparsion of a hadron in terms of its quark and gluon constituents; e.g., for a meson,

$$
|h\rangle=|q \bar{q}\rangle+|q \bar{q} g\rangle+|q \bar{q} q \vec{q}\rangle+\ldots
$$

The various Fock components will mix with each other during their time evolution. However, at sufficiently high hadron energies $E_{h}$, and during short times $t$, the mixing is negligible. Specifical]y, the relative phase $\exp \left[-i\left(E-E_{h}\right) t\right]$ of a given term in $\mathrm{Eq}_{\mathrm{q}}(1)$ is proportional to the energy difference

$$
E-E_{k}=\left[\sum_{i} \frac{m_{i}^{2}+p_{T,}^{2}}{x_{i}}-M_{h}^{2}\right] /\left(2 E_{h}\right)
$$

which vanisbes for $E_{h} \rightarrow \infty$. Hence the time evolution of the Fock expansion is, at high energies, diagonal during the time $\sim 1 / R$ it takes for the hadron to cross a nucleus of radius $R$.

The diagonal time development means that $i t$ is possible to describe the scattering of a hadron in a pucleus in terms of the scattering of its individual Fock components. Let us explore the consequences for typical, soft collisions characterized by momentum trans. fers $q T \simeq \Lambda_{Q C D}$. The partons of a given Fock state will scatter independently of each other if their transverse separation is $\tau \geq 1 / A_{Q C D}$; i.e. if the state is of typical hadronic size. Conversely, the nuclear scattering will be coherent over the partons in Fock states hav. ing $r T<1 / \Lambda_{Q C D}$ since $e^{i t r \cdot r \tau} \simeq 1$. For color-ginglet clusters, the interference between the different parton amplitudes interarting with the nuclear gloonic field is destructive. Thus the nucleus will appear nearly transparent to small, color"singlet Fock states. ${ }^{29}$ In an exper. iment detecting fast secondary hadrons the nucleus in. deed serves, then, as a filter that silects the small Fock components in the incident hadrons.

Consider the intrinsic charm state $|u \bar{d} c \vec{c}\rangle$ of a $\left|\pi^{+}\right\rangle$. Because of the large charin mass $m_{e}$, the energy difference in denominator of the wavefunction will be minimized when the charm quarks have large $I$, i.c. when they carry most of the longitudinal momentum. Moreover, because $m_{e}$ is large, the transverse momenta $p T_{c}$ of the charm quarks range up to $\mathcal{O}\left(m_{c}\right)$, implying that the transverse size of the $c \bar{c}$ system is $\mathcal{O}\left(1 / m_{c}\right)$. Hence, provided only that the $c \bar{c}$ forms a color singlet, it can penelrate the nucleus with litlle energy loss. Thus the high momentum small transverse size $\bar{c} \bar{c}$ color-singlet cluster in the incident hadron passes through the nucleus undeflected, and it can then evolve into charmonium states after transiting the nucleus." In effect, the nucleus is transparent to the beavy quark pair component of the intrinsic state. The remaining cluster of light quarks in the intrinsic charm Fock state is typically of hadronic size and will interact strongly on the front surface of the nucleus. Consequently, the $A$-dependence of the cross section is given by the geometrical factor, $\alpha \simeq 2 / 3$. This justifies the aralysis of Badier et al. $^{24}$ in which the perturbative and non-perturbative charm production mechanisms were separated on the basis of their differenit .4 dependence ( $a=0.97$ and $a=0.77$ for a pion beam, respectively). The effective $\boldsymbol{x}_{\boldsymbol{F}}$-dependence of $\alpha$ seen in charm production is explained by the different charac. teristics of the two production mechanisms. Hard, gluon fusion production dominates at smal $x F$, due to the steeply falling gluon structure function. The contrihution from intrinsic cham Fock stales in the beam peaks at higher $I$, due to the large momentum carried by 
the charm quarks. This two-component hard-scattering plus intrinsic charm model also explains why the buclear dependence of $J / \psi$ produclion depends on $x F$ rather than $2_{2}$, as predicted by leading twist factorization.

An important consequence of this picture is that all final states produced by a penetrating intrinsic $c \bar{c}$ component will have the same $A$-dependence. Thus, in particular, the $\psi(2 S)$ radially excited state will behave in the same way as the $J / \psi$, in spite of its larger size. The nuclcus cannot influence the quark hadronization which (at high energies) takes place outside the nuclear environment.

Quarkonium produstion due to the intrinsic beavy quark state will fall off rapidly for $p T$ greater than $M_{Q}$. reflectiog the fast-falling transverse momentum dependence of the higher Fock state wavefunction. Thus we expect the conventional fusion contrihutions to dominate in the large $p T$ region. The data are in fact consistent with a simple $A^{1}$ law for $J / \psi$ ptoduction at large $P T$. The CERN experiment of Badier et al. ${ }^{24}$ finds that the ratio of nuclear cross sections is close to additive in $A$ for all $x_{F}$ when $p r$ is between 2 and $3 \mathrm{GeV}$. The data of the Fermilab experiment of Katsanevas et al." shows consistency with additivity for $p r$ ranging from 1.2 to 3 GeV.

The probability for intrinsic heavy quark states in a light hadron wave function is expected ${ }^{25,34}$ to scale with the heavy quark mass $M_{Q}$ as $\mathrm{l} / M_{Q}^{z}$. This implies a production cross section propurtional to $1 / M_{Q}^{4}$. The total rate of heavy quark production by the intrinsic mechanism therefore decreases with quark mass, compared to the perturbative cross section which is proportional to $1 / M_{Q}^{2}$. At large $x$ the intrinsic production sbould still dominate, however, implying a nuclear dependence in this region characterized by $\alpha \simeq 0.7 \ldots 0.8$. Experimental measurements of beauty badroproduction in nuclei over the whole range of 2 will be essential for unraveling the two components of the cross section.

\section{SHADOWING AND ANTI-SHADOWING OF NUCLEAR STRUCTURE FUNCTIONS}

The shadowing and anti-shadowing of deep inelastic nuclear structure functions refers to the depletion of the effective number of nucleons $F_{2}^{A} / F_{2}^{N}$ at low $\mathbf{S} 0.1$, and the increase above nucleon additivity at $I \sim 0.15$. Results from the EMC collaboration ${ }^{35}$ and $S L A C^{36}$ indi. cate that the effect is roughly $Q^{2}$-independent; i.e. shadowing is a leading twist in the operator product analysis In comtrast, the shadowing of the real photo-absorption cross section due to p-dominance ${ }^{37-40}$ falls away as an inverse power of $Q^{2}$.

Shadowing is a destructive interference effect which causes a diminished fux and interactions in the interior and back face of the nucleus. The Glauber analysis ${ }^{11}$ corresponds of hadron-nucleus scattering to the follow. ing: the incident badron scatters elastically on a nucleon $N_{1}$ on the front face of the nucleus. At high energies the phase of the amplitude is imaginary. The hadron then propagates through the nucleus to nucleon $N_{2}$ where it interacts inelastically. The accumulated phase of the hadron propagator is also imaginary, so that this twostep amplitude is coherent and opposite in phase to the one-step amplitude where the beam hadron interacts directly on $\mathrm{N}_{\mathbf{2}}$ without initial-stave interactions. Thus the target nucleon $N_{2}$ sees less incoming flux: it is shadowed by elastic interartions on the front face of the nucleus. If the hadron-nucleon cross section is large, then for large $A$ the effective number of nucleons participating in the inelastic interactions is reduced to $\sim A^{2 / 3}$, the numbet of surface nucleons.

In the case of virtual photo-absorption, the photon comverts to a $q \overline{9}$ pair at a distance before the target proportional $10 \omega=x^{-1}=2 p \cdot q / Q^{2}$ in the labora. tory frame." In a physical gauge, such as the light- 
cone $A^{+}=0$ gauge. the final-state itteractions of t]ic quark can be ne'glected in the Bjorken limit, and eflectively only the anti-quark interacts. The nuclear structure function $F_{2}^{A}$ producing quark $q$ can then be written as an integral ${ }^{+3.44}$ ove: the inclastic cross section $\sigma, A\left(s^{\prime}\right)$ wiluere $s^{\prime}$ grows as $1 / x$ for fixed space-like antiquark mass. Tilus the A-dependence of the cross section mimics the $A$-dependence of the $\bar{q}$ cross section in the nucleus. Hung Jung Lu and I have recently applied the standard Glauber multi-scattering theory. to $\sigma_{\bar{\phi}} A$ as. suming that formalism can be taken over to off-shell $\bar{q}$ interactions. ${ }^{45}$ The shadowing mechanism is illustrated in Fig. 4.

(J)

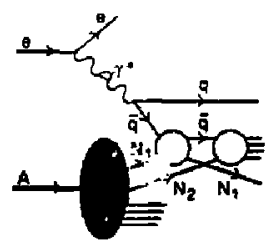

(b)

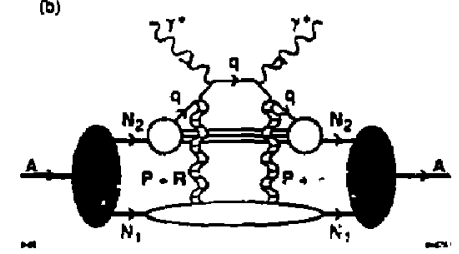

Figure 4. (a) The double-scaltering amplicude that shadows he direct interaction of the anti-quark with $N_{2}$. (b) Th: same mechanism as in (a), drawn in the tradicional "hand-bag" form. Pomeron and Reggeon exchange between the quark line and $N_{1}$ are explisitly illustrated.

Our sesults show that ior reasonable values of the $\bar{q}$-nucleon cross section, one can understand the magnitude of the shadowing effect at small $r$. Moreover, if one introduces an $\alpha_{R} \simeq 1 / 9$ Rrggeon contribution to the $\bar{q} A^{\prime}$ amiplitude, the real phase introduced by such a contribution automatically leads to "anti-shadowing" (effective number of nucleons $\left.F_{2}^{A}\left(x, Q^{2}\right) / F_{2}^{N}\left(x, Q^{2}\right)>A\right)$ at $I \simeq 0.15$ of the tow percont magnitude seen by the SLAC and ESIC" experiments. 35.36

Our analysis provides the input or starting point for the $\log Q^{2}$ evolution of the deep inelastic strunture functions, as given for example by Ifucllet and Qiu. ${ }^{46}$ The parameters for the effective $\bar{q}$ nucleru cross siection required to understand shadouing phenonterta pro. vide important information on the interactions of quarks in nuclear matter. This analysis also has implicalions of the nature of particle production for virtual photo absorption in ruclei. At high $Q^{2}$ and $5>0.3$, hadron production should b. niform throughout the nucleus. At low I where shadowing oceurs, the inelastic reaction occurs mainly at the front surface. These leatures can be examined in detail by studying non-additive multiparticle correlations in both the target and carrent fragmentation region. It should also be emphasized that the same types of multj-scaltering "fan diagrams also appear in the analysis of the saturation of the gluon distribution at small $x$.

The results for the effective number of nucleons $A_{e / f}(x) / A$ are shown in Fig. 5 for $A=12,64$. and 238. One observes shadowing below $x \simeq 0.1$ and an anti-shadowing peak around $r=0.15$. The shadowing effects are roughly logarithmic on the mass number $A$. The magnitude of shadowing predicted by the model is consistent with the data for $x>0,01$; below this region, one expects highrr-twist and vector-meson dominance shadowing to 1 itribute. For $z>0.2$ other nuclear oflects must be taken into account. Most of the parame lecs used in the model are assigned typical hadronic values. The critical quantity is the effective quark-nucleon cross section $\sigma$ which controls the magnitude of shadowing effect near $x=0$; a larger sulue of $\sigma$ implies a larger $\vec{q}^{\circ} N$ cross section and thus more shadowing. Notice that $a$ is the effective cross section at zero $\bar{q}$ virtuality, thus the typical value $\langle\sigma\rangle$ estering the calculation is soruewhat smaller. The magnitude of anti-shadowing 
is determined the real to isnaginary-part satio of the Jeggten scatiering amplitude.

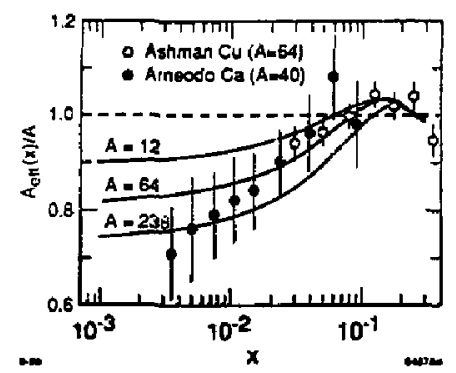

Figure 5 The predicted ratio of $A_{4} f(s) / A$ of the mulit-scattering madel in the low $x$ region for difezent nuclear mass number. The data points are results from the EMC experiment for $\mathrm{Cu}$ and $\mathrm{Ca}$.

Our semi-quantitative analysis shows that parten multiple-scattering process provides a mechanism for explaining the observed shadowing at low $x$ in the EMCSLAC data. The existence of anti-shadowing requires the presence of regions where the real part of the $\bar{q}-N$ amplitude dominates over the imeginary part. The constructive interference which gives anti-shadowing in the $I \sim 0.15$ region is due in this model to the phase of the Reggeon $a=1 / 2$ term. The phase follows from analyticity and is dictated by the shape of the structure functions at low $x$. We could utilize additional terms (at lower values of a) to parameterize other bound-state contributions which vanish as higher powers of $I$, but in practice their qualitative effect would be irdistinguishable from the our simplified model.

The analysis presented here correlates sbadowing phenomena to microscopic quark-nucleon parameters. This approach also provides a dynamical and analytic explanation of anti-shadowing, confirmung the conjecture of Nikolaev and Zakbarov" who preditted that such an effect must exiat on the basis of conservation laws. Using the perturbative QCD factorization theorem for inclusive reactions, the same analysis can be $\mathrm{ex}$ tended to Drell-Yan and ot her fusion processes, taking intc accound the separate dependence on the valence and sea quarks. Thus some shadowing and anti-shadowing should also be observable in the nuclear structure function $F_{2}^{\wedge}\left(I_{2}, Q^{2}\right)$ extracted from massive lepton pair pro duction on nuclear targets at low $\mathbf{x}_{2}$.

\section{TESTS AT JHERA}

Many of the topics I have discussed in this talk can be directly tested at HERA.

1. The QCD model for intrinsic gluons presented in section 2, evolved to HERA momentum transfers. provides detailed predictions for the small $x$ and large $x$ behavior of the gluon and sea quark distributions.

2. Measurements of non-singlet structure functions in charged current reactions and the difference of proton and neutron cross sections using a deuteron beam at HERA will be very important for confirming non-singlet Regge behavior of the leading twist structure functions. This will allow the construrtion of the Regge-iree bound-valence qui--k distributions discussed in section 3 .

3. Studies of shado.ring and anti-shadowing processes on nuclei would be very interesting at HERA energies. Some information can be ob. tained with deuteron beams, but the possibility of heavy ion beams ahould be explored.

4. Diffractive reactions where the proton is tagged in the forward direction lead to funojamental probes of the Pomeron and will give more insight into the multi-gluon matrix elements needed to understand saturation of the gluon structure function." Diffraction of light mass vector meson systems is also important for understanding high energy exclusive reactions and shadowing mecha. 
nisms. Electrofiuduction of the $\pi^{0}$ at high energies would be an ideal way to isolate the Odderon in the $t$-channel, the odd charge conjugation counterpart of the Pomeron predicted by QCD.

5. HERA is the ideal laboratory to confirm or disprove the presence of intrinsic charm and besuty in the proton. Intrinsic heavy quark states can be identified two ways: by flavor-tagging the recoil jet and spectator system in the proton beam direction; and by measuring the longitudinal momentum distribution of heavy quarkoajum in the proton fragmentation region in electron-proton collisions. The presence of charm and beauty in the nucleon at large $x$ would have important implications for our fundamental understanding of hadrons in r.cD.

\section{ACKNOWLEDGEMENTS}

The work reported here was based on collaborations wich Kent Hombostel, Paul Hoyer, Hung Jung Lu, A] Mueller, Chris Pauli, and Ivan Schmidt. This work was supported by the Department of Energy, contract DEAC03-75SF00515.

\section{REFERENCES}

1. K. Hornbostel. S. J. Brodsky, and H.-C. Pauli, Phys. Rev. D41, 3814 (1990); K. Hombostel, SLAC-0333, Ph. D. thesis, unpublished (1988); and M. Burkardt, Nucl. Phys. A504, 762 (1989); and references therein.

2. See, e. g., F. Martin, Phys. Rev. D19, 1382 (1979): M. Glück, E. Reya, and W. Vogelsang, Dortmund University preprint DO-TH-89/3 (1989).

3. S. J. Brodsky and I.A. Schmidt, Phys. Lett. B234, 144 (1990.)

4. V. N. Gribov and L. N. Lipatov, Sov, J. Nucl. Phys. 15, 438 and 675 (1972).
5. J. D. Bjorken, Phys. Rev. Dl, 1376 (1970).

6. See, for example, S. J. Brodsky, J. Ellis, M. Karliner, Phys. Lett. B206, 309 (1988).

7. R. Blankenbecler, S. J. Brodsky Phys, Rev. Dio. 2973 (1974); J. F. Gunion, Phys. Rev. D10, 242 (1974); S. J. Brodsky and J. F. Gunion, Phys. Rev. D19, 1005 (1979), where coherence effects are also discussed.

8. M. Karliper, 24th Kencontse de Moriond, Mar 1989; preprint TAUP 1730-89.

9. P. Aurenche, et al., Phys. Rev. D39, 3275 (1989).

10. See, for example, F. E. Close, An Introduction to Quarts and Partons, Academic Press (1979).

11. J. Kuti and V. F. Weisskopf, Phys. Rev. D4, 3418 (1971).

12. We thank J. Collins for conversations on this point.

13. S. J. Brodsky, F. E. Close and J. F. Gunion. Phy's. Rev. D5, 1384 (1972).

14. W. Weisberger, Phys. Rev. D5, 2600 (1972); S. J. Brodsky, F. E. Close and J. F. Gunion, Phys. Rev. D8, 3678 (1973); S. J. Brodsky and I. Schmidt, unpublished. This result assumes that the valence quark mass only appears in the light-cone kinetic energy $\Sigma\left(k_{1}^{2}+m^{2}\right) / z$.

15. S. J. Brodsky and 1. A. Schmidi, SLAC.PUB.5169 (1990).

16. M. L. Goldberger and F. E. Low, Phys. Rev. 176. 1778 (1968); see also: T. Erber, Ann. Pbys. 6, 319 (1959).

17. We neglect proceses higher order in aQED such as pair production in the electromagnetic ficld of the quark $d$. 
18. For a derivation of the Reggeon contribution to the leading iwist structure functions, see P. $V$. Landshoff, J, C. Polkinghorne and R. Short, Nucl. Phys. B28, 224 (1971), and S. J. Brodsky, F. E Close. and 3. F. Gunion. Phys. Rev. D8, 3678 (1973).

19. ' usual we neglect hadronization effects and other final state interactions at high energies.

20. S. P. K. Tavernier, Rep. Prog. Phys. 50, 1439 (I987): l. Gasparini, Proc. XXIV Int. Conf. on High Energy Physies, (R. Kotthaus and J. H. Kühn, Eds., Springer 1989), p. 971.

21. Detailed predictions for the contribution of intrinsic charm to the nucleon charmed quark structure functions and comparisons with existing leptoproduction dala are given by $E$. Hoffmana and $R$ Moore Z. Phỵs. C20, 71 (1983).

22. M. MacDermott and S. Reucroft. Phys. Lett. 184B, 108 (1987)

23. S. F. Biagi, et al., Z. Phys. C28, 175 (1985). See also P. Coteus, et al. Phys. Rev. Lett. 59, 5030 (1907).

24. J. Badier, et al., Z. Phys. C20, 101 (1983); J. Badier, et al., Phys. Lełt. 104B, 335 (1981).

25. S. J. Brodsky, P. Hoyer, C. Peterson, and N. Sakai, Phys. Lett. 93B, 451 (1980); S. J. Brodsky, C. Pe terson, and N. Sakai, Phys. Rev. D23, 2745 (1981).

26. S. J. Brodsky, J. F. Gunion, and D. E. Soper, Phys. Rev. D36, 2710 (1987).

27. S. J. Brodsky, P. Hoyer Phys. Rev. Lett. 63, 1566 (1989).

28. G. Bertsch, S. J. Brodsky, A. S. Goldbaber, and J. F. Gunion, Phys. Fev. Let2. 47, 297 (1981). In this paper the "color filter" argument was used to predict the production in nuclei of diffrective high mass multi-jet final states with momenturm distributions controlled by the structure of the valence Fock state of the incident hadrons.

29. The diminished interactions of small colorsinglet Fock components also leads to the phenomena of "color transparency" in quasi-elastic badron-nucleon scattering inside of nuclei. Set A. H. Mueller, Proc- XVII Recontre de Moriond (1982), and S. J. Brodsky, Proc. XIII Int. Symp. on Multiparticle Dydamics, Volendam (1982). In this case the dominance of small valence Fock components in large momentum transfer exclusive reacticos implies the absence of initial and final stale interactions. Experimental evidence for color transparency in quasi elasic pp scattering in ouclei at heam momentum up to $10 \mathrm{GeV} / \mathrm{C}$ is given in A. S. Carroll, et. al, Phys. Rev. Leth. 61, 1698 (1988). Explanations for the absence of color transparency at $12 \mathrm{GeV} / \mathrm{c}$ are given in S. J. Brodsky, G. F. De Teramond, Phys. Rev. Lett. 60, 1924 (1988); and in J. P. Ra). ston and B. Pire, Phys. Rev. Lett. 61, 1823 (1988).

30. K. J. Anderson, $d$ al., Phys. Rey. Lett. 42, 944 (1979); A. S. Jo, et al, Phys. Rev. D23, 604 (1981); P. Bordalo, el al., Phys. Lett. 193B, 368 (1987).

31. Yu. M. Antipov, et al., Phys. Lett. 76B, 235 (1978): M. J. Corden, et al., Phys. Lett. 110B, 415 (1982): J. Badier, et al, Z. Phys. C20, 101 (1983); S. Kat. saneves, el al., Phys. Rev. Lett. 60, 2121 (1988).

32. Atternatively, the individual charmed quarks can Iragment into final state charmed badrons either by hadronization or by coalescing with co-moving light quark spectators from the beam. This effect can explain the depletion of the ratio of $J / \psi$ to continuum muon pairs in high transverse energ; 
nucleus-pucleus collisions. See S J. Brodsky and A. H. Mueller, Phys. Lett. B206, 685 (1988).

33. P. Hoyer, M. Vanttinen, and U. Sukbatme, HUTFT-90-11, (1990.)

34. S. J. Brodsky, H. E. Haber, and J. F. Gunjon, jn Anti-pp Options for the Sypercollider, Division of Partjcles and Fjelds Worhshop, Chicago, IL, 1984, edited by J. E. Pilcher and A. R. White (SSCANL Report No. 84/01/13, Argonne, IL, 1984). p. 100. S. J. Brodsky, J. C. Collins, S. D. Ellis, J. F. Gunion, and A. H. Mueller, published in Snowmass Summer Study 1984, p. 227.

35. J. Ashman et w., Phys. Lett. 202B, 603 (1988) and CERR-EP/88-06 (1988)

M. Arneodo ef al., Phys. Lett. 211B, 493 (1988)

36. R. G. Arnold et alPbys. Rev. Let $.52,727$ (1984) and SLAC-PUB-3257 (1983)

37. J. S. Bell, Phy5. Rev. Lett. 13, 57 (1964)

38. L. Stodolsky, Phys. Rev. Lett. 18, 135 (1967)

39. 5. J. Brodsky and J. Pumplin, Phys. Rev. 182,1794 (1969)

40. J. J. Sakurai and D. Schildknecht, Phys. Lett. 40B, 121 (1972); Phys. Lett. 4]B, 489 (1972); Phys. Lett. 42B, 216 (1972)
41. Ћ. J. Glauber, in Lectures in Theoretical Physics. edited by W. E. Brittin at al,(Interscience, Nev: York, 1959), vol. I

42. T. H. Bauer, R. D. Spital, D. R. Yennie and F. M. Pipkin, Rev. Mod. Phys. 50, 261 (1978)

43. P. V. Landshof, J. C. Polkinghorne and R. D. Short, Nucl. Phys. B28, 225 (1971)

44. S. J. Brodsky, F. E. Close and J. F. Gunion, Phys. Rev. D 5, 1394 (1972)

45. S. J. Brodsky, and H. J. Lu, Phys. Rev, Lett $64,1342(1990)$

46. A. H. Mueller and J, Qiu, Nucl. Phys. B268, 427 (1986)

J. Qiu, Nucl. Phys. B291, 746 (1987)

47. See the talks of E. Levin, A. Mueller, and M. Ryskin, this conference.

48. N.N. Nikolaev and V. I. Zakbaroy, MPJ-PAE PTh-11/90, (1990); Physics Letters 55B 397 (1975); Sov. J. Nucl, Pbys. 21, 227 (1975).

49. See M. Ryskin, this conference. 